\title{
ProduCCIÓN Y APROPIACIÓN DEL VALOR EN ARGENTINA: EL ROL DEL DEPRIMIDO SALARIO REAL
}

\author{
Damián Kennedy*
}

Fecha de recepción: 10 de enero de 2013. Fecha de aceptación: 2 de agosto de 2013.

\begin{abstract}
RESUMEN
A partir de una perspectiva tanto histórica como de comparación internacional, el artículo se propone analizar algunas de las características más sobresalientes del proceso de acumulación de capital en Argentina, resaltando lo ocurrido desde mediados de los años setenta. En el marco del estancamiento de la producción de valor, el principal resultado es que el deterioro del ingreso laboral real, observado desde dicho punto de inflexión, tiene la funcionalidad de constituir una fuente extraordinaria de excedente. Tal fenómeno se presenta como una condición necesaria del proceso de acumulación dado el menor dinamismo relativo de la productividad, constituyéndose en un elemento común de los distintos esquemas económicos a partir de allí adoptados, y más allá de otras características de los mismos que puedan juzgarse más o menos beneficiosas.
\end{abstract}

Palabras clave: teorías del valor, producto social, distribución funcional, salario real, productividad.

Clasificación JEL: D31, D46, E24, J24, Y10.

\section{Production and the Appropriation of Value in Argentina: The Role of Real Wage Depression}

\begin{abstract}
From a historical perspective and by international comparison, this article seeks to analyze some of the most noteworthy features of the capital accumulation process in Argentina, emphasizing events since the mid-1960s. In the context of the stagnation of value production, the main outcome has been that impairment to real wages from this inflection point has constituted an extraordinary source of surplus. This phenomenon is a necessary condition for the accumulation process in light of low dynamism relative to productivity, and is a factor common to the different economic schemes that have been adopted, beyond other features that could be judged as more or less beneficial.
\end{abstract}

Key Words: Value theories, social product, functional distribution, real wages, productivity.

* Consejo Nacional de Investigaciones Científicas y Técnicas, Argentina. damian.kennedy@hotmail.com 


\section{PRODUCTION ET APPROPRIATION DE LA VALEUR EN ARGENTINE : LE RÔLE DE} L'ABAISSEMENT DU SALAIRE RÉEL

\section{Résumé}

À partir d'une perspective tant historique que de comparaison internationale, l'article se propose d'analyser quelques unes des caractéristiques les plus notables du processus d'accumulation de capital en Argentine, en mettant l'accent sur ce qui s'est passé depuis le milieu des années soixante. Dans le cadre de la stagnation de la production de valeur, le principal résultat est que la détérioration du revenu réel du travail observé depuis ledit point d'inflexion présente la fonctionnalité de constituer une source extraordinaire d'excédent. Un tel phénomène apparaît comme une condition nécessaire du processus d'accumulation vu le moindre dynamisme relatif de la productivité, et devient donc un élément commun des divers schémas économiques adoptés depuis, au-delà d'autres caractéristiques de ceux-ci susceptibles d'être estimées plus ou moins avantageuses.

Mots clés: théories de la valeur, produit social, distribution fonctionnelles, salaire réel, productivité.

\section{PRODUÇÃO E APROPRIAÇÁO DO VALOR NA ARGENTINA: O PAPEL DO DEPRIMI- DO SALÁRIO REAL}

\section{Resumo}

A partir de uma perspectiva tanta histórica como de comparação internacional, o artigo se propóe analisar algumas das características mais sobressalentes do processo de acumulação de capital na Argentina, ressaltando no ocorrido desde mediados dos anos sessenta. No marco do estancamento da produção de valor, o principal resultado é que a deterioração da renda laboral real observado desde dito ponto de inflexão tem a funcionalidade de construir uma fonte extraordinária de excedente. Tal fenômeno se apresenta como uma condição necessária do processo de acumulaçáo dado o menor dinamismo relativo da produtividade, constituindo-se num elemento comum dos distintos esquemas econômicos a partir daí adotados, e além de outras características dos mesmo que se podem julgar mais ou menos benéficas.

Palavras-chave: teorias do valor, produto social, distribuição funcional, salário real, produtividade.

阿根廷的价值生产和占有：实际工资贬值的影响 摘要:

本文从历史和国际比较视角寻求分析阿根廷资本积累过程的显著特征, 重 点强调了20世纪60年代以来的事件。在价值生产停滞的背景下, 主要后果 是对实际工资的抑制却构成了超额剩余的来源。考虑到生产率相对较低的 动力机制下, 对积累过程而言, 这类现象是一个必要条件, 也是不同经济 计划里的共同因素。与其他特征相比, 可判断它或多或少是有益的。 关键词：价值理论 社会产品功能性分配 实际工资生产率 


\section{INTRODUCCIÓN}

En la literatura que aborda el estudio del proceso de acumulación de capital de Argentina se encuentra un acuerdo relativamente generalizado respecto tanto a sus etapas en el último tercio de siglo, como de las características centrales de cada una de ellas. Así, como consecuencia de la "crisis de los treinta" y, particularmente, de la segunda guerra mundial, se estableció un modelo de industrialización por sustitución de importaciones (ISI), el cual, interrumpido abruptamente por el golpe militar de 1976, fue remplazado por una "apertura con endeudamiento externo" o "valorización financiera", con particular vigencia durante el gobierno militar (1976-1983) y el régimen de convertibilidad (1991-2001) (Basualdo, 2006; Diamand, 1972; Ferrer, 2004, y Schvarzer y Tavosnaska, 2008). Finalmente, luego del estallido de la convertibilidad, con una profunda devaluación en el inter, la adopción de un tipo de cambio real "competitivo y estable" generó un patrón de crecimiento de características similares a la ISI, basado en la producción de bienes industriales con fuerte absorción de fuerza de trabajo (Arceo et al., 2007; Basualdo, 2006, y Frenkel y Rapetti, 2004).

El estudio de la distribución funcional del ingreso no ha sido ajeno a dichas etapas. Habiendo ocupado un papel clave en el marco de la ISI, ha sido abandonado progresivamente desde su finalización, para prácticamente desaparecer en la década de los noventa, en el marco de una creciente ausencia de información al respecto (Lindenboim et al., 2005). En los últimos años comenzó a revertirse dicha tendencia, marco en el cual se inscribe nuestra investigación, que se inició con la estimación de la serie para el lapso 19932004 (Lindenboim et al., 2005), luego ampliada para 1950-2006 (Grańa y Kennedy, 2008b) y 1935-2010 (Kennedy, 2012), ${ }^{1}$ y que ha estudiado distintos aspectos parciales de ésta para el lapso 1950-2006 (Lindenboim et al., 2010).

La conclusión más importante, en lo que respecta al presente artículo, es que la caída de la participación asalariada desde mediados de la década de los setenta, se explica por la conjunción de incrementos de productividad y deterioro del salario real, en contraposición a lo verificado en los países re-

1 Todo lo que las estadísticas argentinas informen desde el año 2007 debe ser tomado con suma cautela, dada la intervención que sufrió el Instituto Nacional de Estadísticas y Censos (INDEC) a inicios de 2007. En los referidos trabajos pueden encontrarse los procedimientos metodológicos adoptados, incluyendo el tratamiento especial de la información en cuestión desde dicho año. 
conocidos como "desarrollados" (esto es, países en los cuales las leyes de la producción capitalista tienden a presentarse de manera relativamente directa -o inmediata-), donde se observa, desde el mismo momento, un deterioro tendencial de dicha participación (de menor magnitud y variabilidad que la argentina), aunque con un incremento de productividad de mayor proporción que el del -también creciente- salario real (Kennedy y Grańa, 2010).

¿Qué papel desempeña en el proceso de acumulación de capital de nuestro país dicho deterioro del salario real? La respuesta a esta pregunta depende, en primera instancia, del contenido conceptual que se asigne a las variables salario real y productividad. Ahora bien, en tanto la distribución funcional del ingreso es una de las facetas del producto social, en rigor la problemática se extiende "un paso atrás": la respuesta dependerá, en definitiva, del concepto de producto social que se sostenga, aspecto que constituye, en consecuencia, el punto de partida del presente artículo.

De esta forma, se proponen aquí dos objetivos fundamentales: en primer lugar, desarrollar conceptual y empíricamente una forma alternativa de presentar la evolución económica de Argentina, que considere no sólo el carácter de valor de uso del producto social (captado por el producto a precios constantes) sino también la evolución de su carácter de valor. En segundo lugar, poner de manifiesto el papel de fuente extraordinaria de excedente del deterioro del salario real, como consecuencia del estancamiento de largo plazo de la masa de valor anual.

Así, en la primera sección, sintetizamos la discusión en torno al doble carácter del producto social anual de un determinado país, presentando su evolución para Argentina para el lapso 1935-2010, marco en el cual, en la segunda, repasamos el devenir de la participación asalariada y de sus componentes -productividad y salario real-, como faceta del producto social que vincula al proceso de producción con la participación económica de la población. En ambos casos, contraponemos los resultados con los de Estados Unidos, país que tomamos como representante de los países reconocidos como desarrollados. Por su parte, en la tercera sección discutimos el deterioro del ingreso laboral real como fuente extraordinaria de plusvalor, presentando una cuantificación del fenómeno, base sobre la cual discutimos algunos aspectos del acuerdo respecto del proceso de acumulación de Argentina; se resalta la posconvertibilidad.

Como quedará de manifiesto, este artículo reviste un carácter general el cual, atendiendo a las restricciones de espacio, se escogió intencionalmente. Y lo es, en tanto procura sintetizar la unidad de una línea de investigación que comienza en los rasgos generales del capitalismo como relación social univer- 
sal de organizar la producción y avanza hasta sus formas concretas de manifestarse en nuestro país, con el objetivo de, enfocados en algunas de tales formas, explicar ciertos pilares estructurales de su proceso de acumulación (más allá de que puedan serlo de procesos inmediatos con mejores o peores consecuencias para el conjunto de su población).

\section{CONTENIDO Y EVOLUCIÓN DEL DOBLE CARÁCTER DEL PRODUCTO SOCIAL ${ }^{2}$}

\section{La especificidad del producto social}

Para las Cuentas Nacionales, el producto social anual de un país constituye una masa de valores de uso, donde el precio aparece como el elemento natural común con mayores ventajas para su agregación (y, por ello, es por lo que debe dejarse "constante" para el cómputo de la evolución de aquél) (Monteverde y Sallaberry, 1981; Müller, 1998; Naciones Unidas, 1993, y Propatto, 2004). Estas conceptualizaciones se sustentan en los fundamentos básicos de nuestra ciencia, aparentemente comunes a toda concepción teórica (o independientes de cualquiera de ellas). ${ }^{3}$

Ahora bien, en rigor, tal noción de producto tiene su origen en el marginalismo, toda vez que dicha escuela de pensamiento lo considera unilateralmente como un "cúmulo de cosas" escasas (útiles y limitadas en cantidad), que explica tanto la esencia como la magnitud del valor de cambio. Así, la determinación de ambos atributos resulta un fenómeno natural, explícitamente desvinculado de la organización social del proceso de trabajo humano productor de valores de uso (Walras, 1987 [1874]).

En contraposición, la economía política clásica (EPC) considera al trabajo como la fuente de la riqueza, de modo que la magnitud de valor de cambio aparece determinada por la cantidad de trabajo social que cuesta producir los valores de uso (Ricardo, 2004 [1817], y Smith, 1997 [1776]). Sobre esta base, Ricardo distingue para el conjunto de la producción social entre riqueza y valor, toda vez que, en medio de dos momentos del tiempo, idéntica masa

2 El presente apartado constituye una síntesis de algunas temáticas planteadas en Kennedy (2012).

3 Es habitual que se proponga una vinculación con la teoría keynesiana (Propatto, 2004), aunque, en rigor la misma está relacionada con la identificación de los agregados macroeconómicos, pero no con la discusión respecto de qué es el producto, del cual Keynes no presenta una mirada específica. 
de trabajo rinde idéntico valor, aunque puede resultar en un cúmulo distinto de bienes. Entonces, dada la inexistencia de una medida invariable del valor, para dar cuenta de la evolución del mismo propone al trabajo mismo, como si dicho contenido pudiera expresarse directamente como tal (Ricardo, 2004 [1817]). ${ }^{4}$ El problema reside en que para la EPC la esencia del valor aparece (igual que en el marginalismo) como independiente de la forma de organización social del proceso de trabajo.

Es Marx (1995 [1867]) quien plantea que los productos del trabajo portan el atributo del valor de cambio como reflejo de la forma específica que la división social del trabajo tiene en el capitalismo: el carácter privado del trabajo. Esto implica que la organización de la producción social no se realiza bajo relaciones sociales directas, sino mediante el carácter de valor de los productos del trabajo, esto es, de su capacidad de expresar la relación social que sus productores no pueden manifestar directamente. Ahora bien, por el propio carácter privado del trabajo, el valor no puede aparecer directamente como tal, sino en la relación de unas mercancías con otras, como valor de cambio, cuya expresión simultánea para el conjunto de los fragmentos privados de trabajo ocurre en la mercancía dineraria. Así, el precio no es un elemento material de los valores de uso, sino su forma social de presentarse en el capitalismo.

De esta manera, podemos completar aquella "doble" idea de Ricardo, concluyendo en el doble carácter del producto anual de una sociedad: de un lado, constituye un cúmulo de valores de uso (o producto material); del otro, atendiendo a su forma específicamente capitalista, es una masa de valor. Es decir, se trata de extender para el conjunto de las mercancías la idea según la cual "la mercancía como valor de uso satisface una determinada necesidad y constituye un elemento específico de la riqueza material. En cambio, el valor de la mercancía mide el grado de su fuerza de atracción sobre todos los elementos de la riqueza material. Mide, por tanto, la riqueza social de su poseedor" (Marx, 1995 [1867]: 90). Como tal, dicha masa de valor se presenta en lo concreto bajo la forma de masa de valor de cambio o de precio, la cual no refleja en términos cuantitativos inmediatamente la cantidad de trabajo social encerrada en la mercancía, ${ }^{5}$ aunque sí la "riqueza social de su poseedor", en

4 Este resultado sirve de base, no pocas veces, para que desde una perspectiva marxista se proponga como medida de la riqueza las horas de trabajo invertidas en la producción (Shaikh y Tonak, 1994).

5 Subyacen aquí las discusiones no saldadas en la literatura respecto al intercambio de las mercancías por sus valores y, más complejamente, a la transformación de los valores en precios de producción, de las cuales hemos presentado nuestra perspectiva en Kennedy (2012). Si bien 
nuestro caso, un país. En este contexto, y sin perjuicio de lo anterior, por simplicidad de la exposición en lo que sigue del presente artículo, nos referiremos al carácter de valor del producto social.

\section{La evolución del carácter de valor del producto social}

Si se toma un año en particular, resulta imposible distinguir entre el carácter de masa de valores de uso y del producto social, en tanto la homogeneización de los bienes y servicios necesariamente ocurre por su precio. Así, es en la evolución entre años donde se pone verdaderamente de manifiesto su doble carácter. La medición a precios constantes da cuenta justamente de la evolución del carácter de valor de uso. Por lo planteado previamente, para dar cuenta de la evolución de su carácter de valor el foco debe inicialmente estar puesto en la evolución de la capacidad del dinero de representar valor, dado que el mismo no constituye su medida invariable.

Ahora bien, en tanto en lo concreto el dinero es remplazado por signos de valor, el foco se traslada a la evolución de éstos en su capacidad de representarlo. ${ }^{6}$ Esta cuestión nos pone frente a la problemática de que, teniendo la organización de la producción social en el capitalismo un carácter universal, mundial (Iñigo, 2004, y Marx, 2005 [1857-1858]), no hay un signo de valor de tal alcance, ya que cumple su función a partir del curso forzoso que le dan los estados nacionales (Marx, 1995 [1867]).

Para fines expositivos, consideremos inicialmente la presencia de un signo de valor mundial. En tales circunstancias, éste modifica su capacidad de representar valor a causa de dos fuentes: la evolución de la productividad en la producción de la mercancía dineraria y la relación entre la masa de signos de valor lanzados a la circulación y la mercancía dineraria que en su ausencia circularía. Estas determinaciones no son identificables directa e independientemente, son de utilidad para fijar criterios de aplicación práctica a los fines propuestos: la primera determinación es captada a partir de la evolución de la capacidad productiva del trabajo en general, mientras que la segunda lo es por la evolución del precio de una canasta de valores de uso de composición constante.

En este contexto, hay que avanzar sobre la evolución de la capacidad de representar el valor de las monedas nacionales, la mayoría de las cuales (entre

revisten un particular interés en diversos campos, es de esperar que no alteren la esencia de lo aquí planteado, dado que nuestro foco está puesto en las tendencias de largo plazo de la evolución del carácter de valor del producto social.

6 Partimos aquí de las ideas generales presentadas en Iñigo Carrera (2007: 32-34). 
ellas, el peso argentino) no son reconocidos directamente como tales en el mercado mundial, papel que ha desempeñado por excelencia el dólar estadounidense. Así, se consideran las condiciones de producción de Estados Unidos como las sociales medias de producción y, por tanto, las que determinan, en términos generales, el valor de las mercancías.

De esta forma, el dólar no modifica su capacidad de representar valor por la primera de las fuentes (evitando así la problemática de que no necesariamente hay una relación inversamente lineal entre productividad y valor), en tanto que el peso nacional lo hace en función de la evolución de la capacidad productiva del trabajo relativa a Estados Unidos. En cambio, la segunda fuente (captada específicamente por la evolución del IPC) es de contenido nacional, de modo que la determinación corresponde a ambos países. ${ }^{7}$ La evolución del carácter de valor del producto social surge de deflactar los precios de las mercancías por el cociente entre la evolución de la productividad estadounidense y la argentina, y por el IPC. La siguiente expresión sintetiza el cómputo en cuestión:

$$
\text { Evolución valor }=\text { Producto precios constantes } \star \frac{|P|}{\frac{\text { Evol. productividad EU }}{\text { Evolución productividad Argentina }} * \mathbb{P C} \text { Argentina }} * 100
$$

\section{El doble carácter del producto social en Argentina (1935-2010) ${ }^{8}$}

Como puede verse en la gráfica 1 , la evolución del producto a precios constantes en Argentina muestra desde 1935 hasta mediados de los años setenta

7 Podrían considerarse otros indicadores para la captación de las dos fuentes de modificación de la capacidad de la moneda de representar valor. En cuanto al IPC, en general se lo considera el más apropiado para captar la evolución del poder adquisitivo de la moneda. En relación con la productividad, consideramos aquí la del total de la economía, en tanto los resultados reflejarán la situación mínima, al incluirse sectores (como el público) que compensan el mayor dinamismo de los países desarrollados en el resto. En cualquier caso, en Kennedy (2012) hemos presentado los resultados de tomar la productividad industrial, así como también de considerar a Alemania y Japón como países determinantes del valor de las mercancías, con resultados similares a los que aquí se presentan.

8 Aquí se describirá la evolución del mismo en comparación con el estadounidense, dejando para las conclusiones una posible explicación de la misma. Se presenta el debate respecto de lo apropiado de realizar comparaciones internacionales del producto social, dado el creciente proceso de diferenciación de la producción. En el marco del presente artículo, en lo que toca al carácter de valor de uso del producto social la cuestión aparece relativizada en tanto el foco está puesto en su evolución, mientras que en relación con su otra faceta no se presenta inconveniente, toda vez que se trata justamente de captar la capacidad de producción de riqueza abstracta. 
Gráfica 1. PIB a precios de mercados constantes y PIB e IPI en moneda con capacidad de representar valor constante (productividad total de la economía en relación a Estados Unidos e IPC). Argentina y Estados Unidos, 1935-2010. $1935=100$.

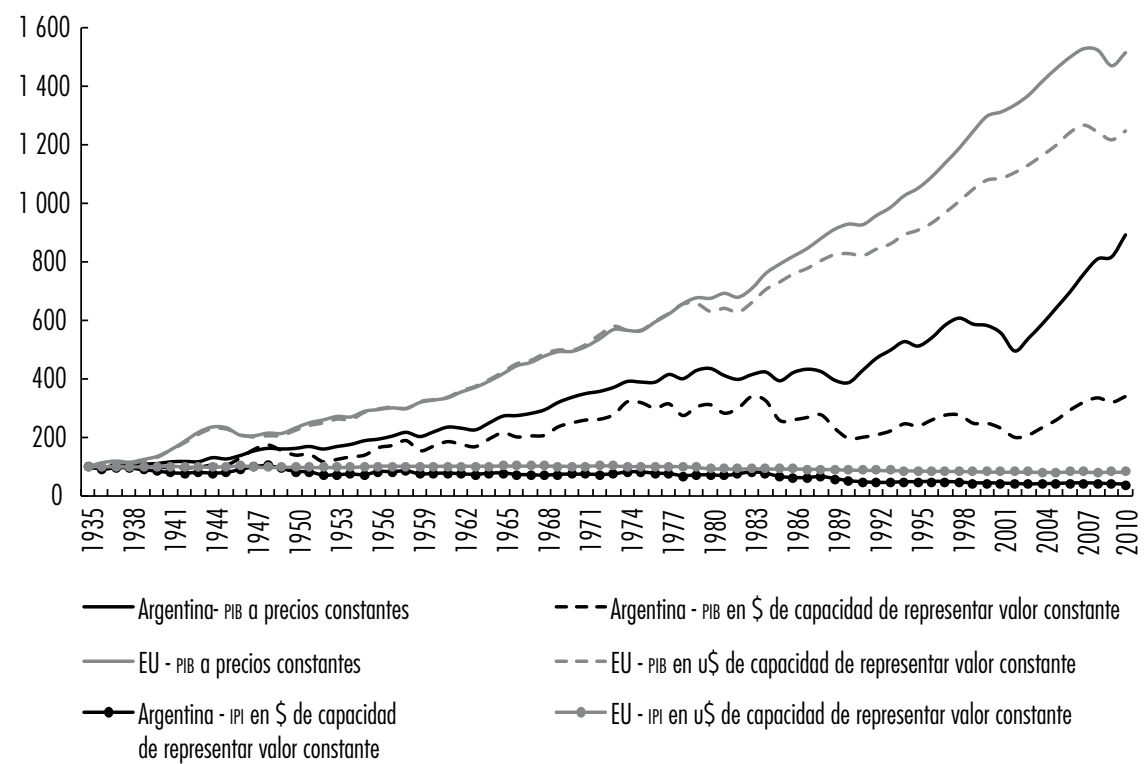

Fuente: Elaboración propia sobre la base de SAE (1955), BCRA (1975), CEPAL (1988, 1991 y 2010), EPH, Cuentas Nacionales e IPC (Indec), Llach y Sánchez (1984), Ferreres (2005), Lindenboim et al. (2005), Iñigo Carrera (2007), Graña y Lavopa (2008), Graña y Kennedy (2008b), CIFRA (2012), BEA y BLS (EU).

un crecimiento permanente, más allá de las interrupciones de los ciclos de stop and go propios de la IsI. A partir de allí, bajo el gobierno de facto y el primer gobierno democrático (1983-1989), éste entra en un periodo de recurrentes oscilaciones pero, sobre todo, de un particular estancamiento, lo que le valió a los ańos ochenta el mote de "década perdida".

La década siguiente estuvo signada por el plan de convertibilidad, ${ }^{9}$ en cuya primera etapa presenta una marcada tendencia positiva, revertida parcialmen-

9 El mismo fue implementado para hacer frente a la profunda hiperinflación de 1989 y 1990. A pesar de que habitualmente se señala que el control de la inflación ocurrió por la conversión directa de la moneda en sí, en rigor aquello ocurrió gracias a la sobrevaluación de la moneda nacional. 
te hacia sus últimos años, luego de cuatro años de recesión y una caída sin precedentes en 2002, en el marco de una creciente restricción al endeudamiento externo que culmina en una violenta devaluación que marca el fin de dicho plan. A partir de allí el mismo ingresa en una fase de crecimiento elevado y sostenido (excepto 2009), lo que lleva a postular la presencia de un nuevo patrón de acumulación. Así, hacia 2010 la producción material resulta más del doble de la observada a mediados de los años setenta.

Ahora bien, la evolución del carácter de valor del producto social proyecta una imagen marcadamente diferente. Ya en los años cincuenta comienza a evidenciarse una evolución más débil en relación al producto a precios constantes, generándose una brecha que se mantiene hasta la década de los ochenta. Pero lo verdaderamente importante es que hacia finales de esa y principios de la siguiente la masa de valor anual se derrumba $33 \%$, pozo al que se retorna con el estallido de la convertibilidad en 2002. El crecimiento posterior, aunque menor al del producto a precios constantes, es importante, lo que arroja como resultado que hacia finales de la década la masa de valor anual argentina presenta un nivel similar al de mediados de los años setenta. ${ }^{10}$

En comparación con Estados Unidos, puede observarse que su producto a precios constantes también muestra un mayor dinamismo que su carácter de valor, ${ }^{11}$ aunque respecto a nuestro país la diferencia en la evolución de este último es marcadamente mayor. Así, mientras que la brecha de la producción material se expandió 40\% desde 1935 hasta la vigencia de la IsI y $70 \%$ hasta 2010, el carácter de valor lo hizo, respectivamente, en 1.8 y 3.7 veces.

En resumidas cuentas, lo que encontramos es que mientras que el producto a precios constantes de Argentina muestra una tendencia de largo plazo creciente (con momentos de profundos retrocesos pero con otros de particular vigor), el carácter de valor de esta producción, su "poder social", se encuentra estancado desde mediados de la década de los setenta, cuando entre 1935 y dicho momento había crecido sistemáticamente, de modo similar relativamente al producto a precios constantes.

10 Como resultado de lo anterior, el valor individual de las mercancías, tal como indica la serie del IPI argentino en moneda con capacidad de representar valor constante, retrocede "entre puntas" 60 por ciento.

11 Tal como se observa en la misma gráfica 1 , el valor individual de las mercancías producidas por Estados Unidos retrocede en "apenas" 20 por ciento. 


\section{DOBLE CARÁCTER EL PROCESO PRODUCTIVO Y EVOLUCIÓN DE SUS VARIABLES COMPONENTES}

\section{El producto social como una masa de valor que encierra plusvalor ${ }^{12}$}

Si trasladamos la mirada al proceso productivo, éste debe dar cuenta del doble carácter de la mercancía y, por tanto, del producto social. En su aspecto genérico, se trata de un proceso productor de valores de uso, donde los elementos que intervienen interesan según su función material, esto es, trabajo, medio de trabajo y objeto de trabajo (los "factores de la producción" de la economía marginalista). En su aspecto social, por su parte, constituye un proceso de producción de plusvalor, en el cual aquellos elementos que participan desempeñan un papel específico: los medios de producción entran como trabajo materializado (cuyo valor se transfiere al del producto), en tanto que el trabajo lo hace como creador de valor. De la diferencia entre este valor y el encerrado en el de la fuerza de trabajo (el valor de medios de vida cuyo consumo implica la presencia de cada fuerza de trabajo con sus atributos productivos) brota el plusvalor. En consecuencia, el producto social en rigor tiene la forma específica de una masa de valor que encierra -hasta aquí- dos componentes: masa salarial y plusvalor. ${ }^{13}$

En este nivel de abstracción, las magnitudes de ambos agregados coincidirían cuantitativamente con las categorías de las Cuentas Nacionales de Retribución Bruta al Trabajo Asalariado y el Excedente Bruto de Explotación (Shaikh, 1984). Ahora bien, debemos realizar al respecto dos consideraciones de importancia: en primer lugar, aquella vinculada a la problemática del trabajo improductivo, pues al no crear valor, ${ }^{14}$ su sostenimiento (tanto en lo que

Para logar la apropiación creciente del medio, una parte del trabajo social desplegado en un ciclo productivo debe cobrar la forma no sólo de medios de vida, sino también de medios de producción. Consecuentemente, dada la presencia de la fuerza de trabajo como mercancía, la separación material anterior tiene la forma de la separación del valor creado en masa salarial y plusvalor. La mayor parte de los planteos críticos del capitalismo considera que tal determinación está portada en la voluntad individual de los capitalistas, no distinguiendo entre el contenido objetivo del proceso con el fin subjetivo del capitalista (Marx, 1995 [1867]).

Se trata de una problemática particularmente compleja, sin acuerdo unánime en la literatura. En nuestra opinión, la raíz de la cuestión reside en que el hecho de que en el capitalismo los valores de uso no son reconocidos directamente como sociales implica, a su vez, que una parte del tiempo de trabajo de la sociedad debe necesariamente destinarse a la realización de las mercancías. Al no crear ni transformar valores de uso, se trata de un trabajo que no crea 
corresponde a la masa salarial como a la ganancia) proviene de la plusvalía producida por el trabajo productivo, ${ }^{15}$ constituyéndose en un "gasto" de la circulación (Marx, 2000 [1885]). En tanto esta distinción resulta en la práctica dificultosa (Müller, 1998), la plusvalía destinada al pago de los salarios correspondientes al trabajo improductivo no puede identificarse como tal; así, la plusvalía se presenta directamente como "neta de gastos de circulación", a la que nos referiremos, por simplicidad expositiva, con la categoría simple de plusvalía.

En segundo lugar, hay que tener en cuenta que los ingresos tanto de los cuentapropistas como de los patrones constituyen ingresos atribuibles al trabajo (Kennedy, 2012), que deben considerarse de forma separada a la plusvalía. En este sentido, y a diferencia de lo realizado en trabajos previos, se dirigirá aquí no al salario sino al ingreso laboral, más allá de que en algunos casos, por conveniencia expositiva, se continúe utilizando la denominación "salario" o "masa salarial".

\section{Distribución funcional del ingreso en Argentina en perspectiva histórica}

La forma habitual de analizar la distribución funcional es aquella que observa la participación de la masa salarial en el ingreso total generado, que se expresa en la gráfica $2 .{ }^{16}$ En el marco del presente artículo, el rasgo fundamental a señalar es que aquí también la irrupción de la dictadura militar implicó un profundo quiebre: mientras que bajo la IsI "en funcionamiento" - desde 1945- la participación de la masa de ingresos laborales representaba entre 55 y 60\% del ingreso total, desde mediados de los años setenta dicho nivel se encuentra entre 45 y 50\% del mismo. Cierto es que en Estados Unidos desde mediados

valor y es, por tanto, improductivo (Marx 2000 [1885]), lo cual no implica que sea innecesario (Fernández Enguita,1985; Guerrero, 1990; Shaikh, 1984).

15 Justamente por esta razón, y en contraposición al planteo de Shaikh (1984) y Shaikh y Tonak (1994), la masa de valor anual no se ve alterada.

16 Para el cómputo de la masa de ingresos de patrones y cuentapropistas en el caso argentino, para el lapso1993-2010 se consideró idéntica metodología que la utilizada para la masa salarial (Graña y Kennedy, 2008a , y Kennedy, 2012), luego empalmada a partir de imputarle al conjunto de patrones y cuenta propia el mismo ingreso que el promedio de los asalariados. Es importante destacar que para el lapso 1993-2010 la evolución del ingreso de cuentapropistas y patrones es similar a la del salario promedio. Para el caso de Estados Unidos, se aplicó el ingreso promedio de los asalariados al total de ocupados no asalariados para el conjunto del periodo. 
Gráfica 2. Masa salarial, ingreso de los cuentapropistas y patrones e ingreso laboral. Participación en el PIB a precios básicos. Total de la economía. Argentina y EU, 1935-2010 (porcentaje).

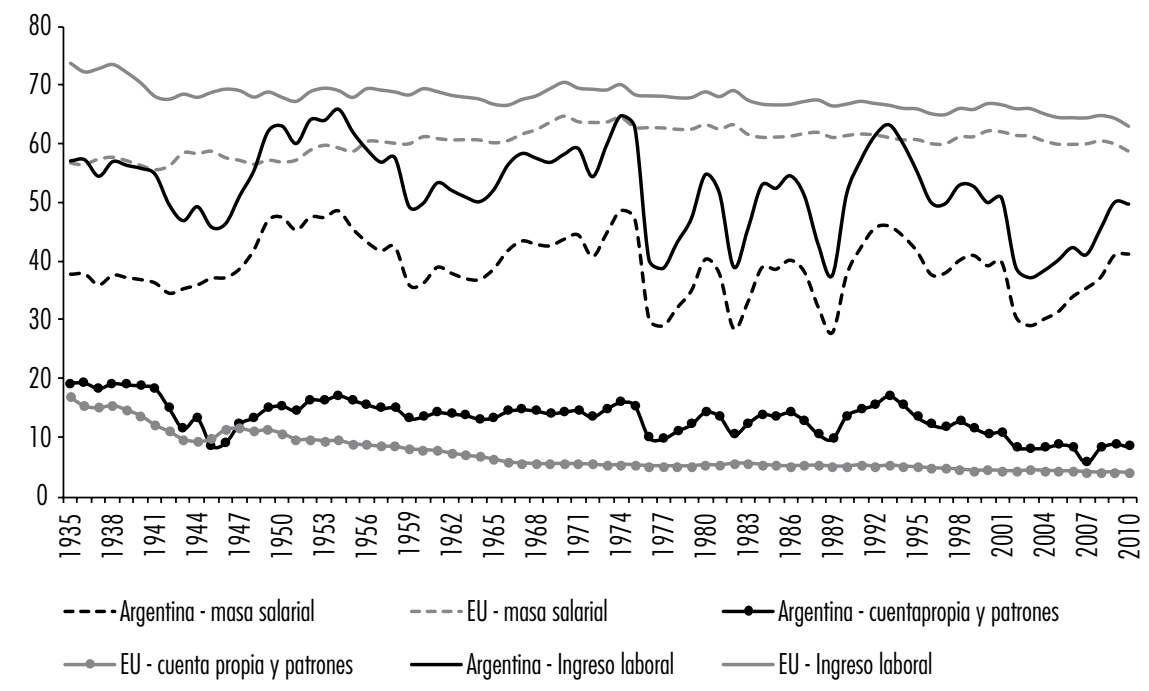

Fuente: Elaboración propia sobre la base de SAE (1955), BCRA (1975), CEPAL (1988, 1991 y 2010), EPH y Cuentas Nacionales (Indec), Llach y Sánchez (1984), Graña y Lavopa (2008), Graña y Kennedy (2008a), CIFRA (2012), BEA y BLS (EU).

de los setenta se observa una tendencia decreciente de la participación de los ingresos laborales en el ingreso total; ahora bien, siempre en un contexto en el cual dicha participación es marcadamente mayor a la argentina (entre 65/70\% del ingreso total), esta caída es tanto de menor magnitud como de carácter paulatino, en contraposición a la de Argentina, donde a partir de mediados de los setenta la mayor frecuencia y magnitud de los cambios de sentido se profundizó notablemente.

Más específicamente, en nuestro país se observan recurrentemente un mínimo de $40 \%$ del ingreso total, "pozo" al cual se llega por primera vez tras el auténtico derrumbe de 25 puntos porcentuales (pp) de comienzos de la dictadura, y que se retorna como consecuencia de la crisis de la deuda externa (1982), la hiperinflación (1989-1990) y el estallido del régimen de convertibilidad (2002). En este contexto, el crecimiento de la última década, aunque importante, alcanzó hacia 2010 para recuperarse de la debacle de la salida de la convertibilidad, aunque no para llegar al "techo neoliberal".

De esta forma, considerando conjuntamente esta dinámica de la participación de los componentes del ingreso con los resultados expresados en la prime- 
ra sección, es posible concluir que desde mediados de la década de los setenta la masa de ingresos laborales se ha deteriorado absolutamente en términos de valor, ocurriendo lo contrario con la de plusvalía, toda vez que el carácter de valor del ingreso total se encuentra estancado. Este retroceso de la masa de valor, representada por los ingresos laborales, no se explica por una menor cantidad de ocupados: tal como puede verse en la gráfica 3, la ocupación presenta una tendencia de largo plazo manifiestamente creciente, incluso no tan diferente (al menos con relación al resto de las variables) a la de Estados Unidos, aunque claramente más inestable, resultado de los ciclos económicos reseñados.

En consecuencia, y como puede verse en la misma gráfica, tanto la masa de valor como el ingreso laboral en valor por ocupado desde mediados de los años setenta se contrajeron profundamente, en contraposición a lo mostrado tanto por Argentina durante la IsI como por Estados Unidos a lo largo de la totalidad del periodo, más allá de que en este último país desde mediados de los setenta decrece su ritmo de crecimiento, a la vez que en el periodo previo es más elevado que en Argentina. Para continuar avanzando debemos introducirnos en las tendencias encerradas en la producción de plusvalía relativa.

Gráfica 3. Ocupados, ingreso laboral y PIB por ocupado en moneda con capacidad de representar valor constante. Argentina y Estados Unidos, 1935-2010. Evolución (1935 = 100).

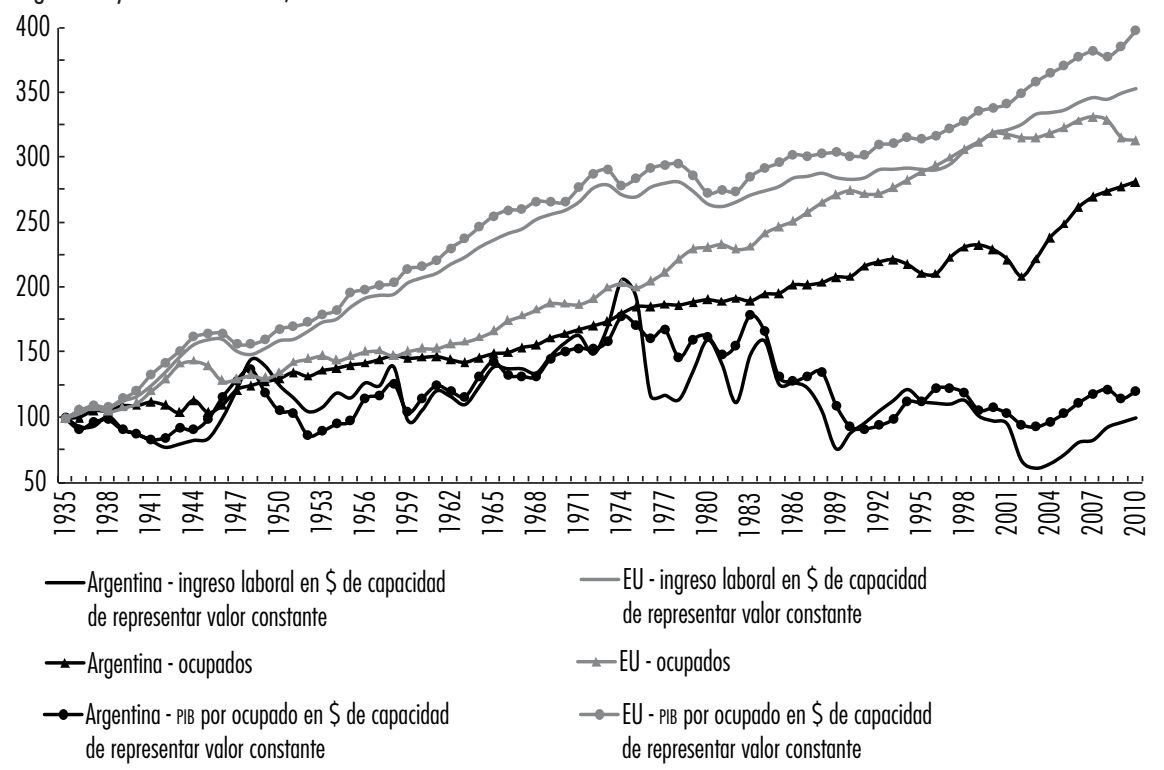

Fuente: idem, gráfica 1. 


\section{La evolución de la productividad y el salario real}

El objetivo inmediato de la producción social en el capitalismo es la expansión de la plusvalía, cuya forma más potente ocurre con la producción de plusvalía relativa (dados los conocidos límites en la producción de plusvalía absoluta), consecuencia del desarrollo constante de las fuerzas productivas del trabajo social: el incremento de la productividad reduce el valor de la generalidad de los valores de uso, de modo que, en cuanto toca a los medios de vida encerrados en la fuerza de trabajo, reduce -a un nivel dado del poder adquisitivo del salario- el valor de ésta, incrementándose la porción de la jornada de trabajo que cobra la forma de plusvalor (Marx, 1995 [1867]). Ahora bien, como tal incremento productivo requiere, en término medio, una creciente calificación del conjunto de la fuerza de trabajo, ${ }^{17}$ la capacidad de consumo de la misma debe aumentar al crecer el valor que porta su fuerza de trabajo. De esta forma, es de esperar que una mayor productividad vaya acompañada de incrementos en el salario real.

Como puede verse en la gráfica 4, bajo la isi la productividad argentina muestra una tendencia creciente, y de ritmo similar a la estadounidense. Este comportamiento se modifica de manera radical, nuevamente, con la irrupción de la dictadura militar, momento a partir del cual pueden identificarse cuatro ciclos diferentes. Los primeros 15 años son de estancamiento y retroceso, resultado del estancamiento del producto material y el crecimiento de la ocupación ya observados. Luego, a partir de la implantación del plan de convertibilidad se evidencia una profunda recuperación, claro que como reflejo de la destrucción de los capitales de menor productividad (resultado de la sobrevaluación cambiaria en conjunción con la apertura comercial).

Sin embargo, la propia razón del éxito es la que marca los límites de esta expansión: modificada la estructura productiva en el sentido expuesto, la productividad entró en una fase de sostenido estancamiento, seguido del retroceso como expresión del fin de la convertibilidad. Finalmente, en el periodo de la postconvertibilidad se observa una importante y genuina expansión de la capacidad productiva del trabajo, que efectivamente "recuerda" a la IsI, toda vez que ocurre en un marco de crecimiento tanto del producto material como del empleo. Sin embargo, no debe perderse de vista que este proceso reciente no sólo no alcanza para cerrar la brecha que se abrió con la (siempre creciente) productividad estadounidense desde principios de la serie, sino que tampoco lo logra respecto de su profundización desde mediados de los años setenta.

17 Un extenso desarrollo de esta determinación puede encontrarse en Graña (2013). 
Gráfica 4. Productividad e ingreso laboral real. Total de la economía. Argentina y EU. 1935-2010. Evolución, $(1935=100)$.

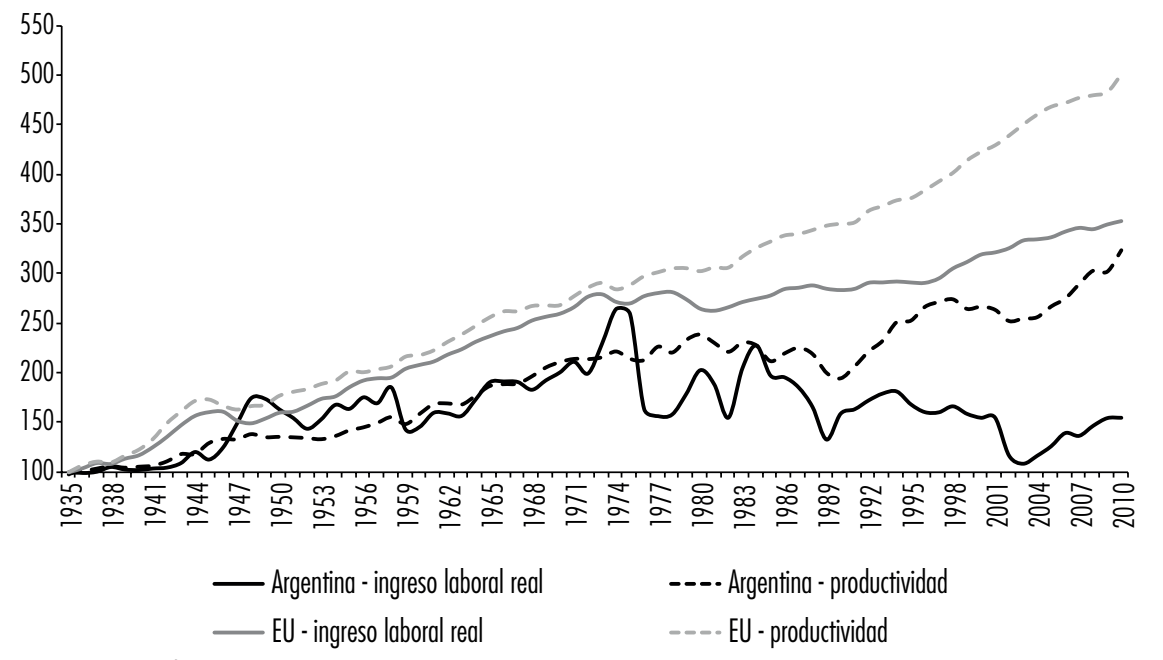

Fuente: idem, gráfica 1.

En la misma gráfica 4 se expresa la evolución del ingreso laboral real. En el caso argentino, su evolución a lo largo de la ISI es positiva, duplicándose entre 1935 y los primeros años de la década de los setenta, mostrando un comportamiento similar al de Estados Unidos. El marcado y particular crecimiento entre 1972 y 1974 es el preludio de un derrumbe sin precedentes: la irrupción de la dictadura militar se lleva consigo mucho más que esta última expansión. Así, hacia 1977 el poder adquisitivo del ingreso laboral representa 75\% del vigente a inicios de los ańos setenta, ${ }^{18}$ nivel que, lejos de constituir un mínimo histórico, reaparece recurrentemente en los momentos más críticos del proceso económico nacional.

Más específicamente, en primera instancia retorna a dicho nivel con la crisis de la deuda de 1982, luego de una recuperación parcial durante la dictadura. Después, bajo el gobierno democrático pueden verse apenas dos años de recuperación, seguidos de una sostenida disminución que, mediante hiperinflación, desemboca en un ingreso laboral real que representa $66 \%$ del

18 Elegimos tal momento para la comparación pues hasta allí el salario real presenta una evolución acorde a la evolución de la capacidad productiva del trabajo. Evidentemente, si tomáramos el nivel de 1973-1974, las tendencias aquí señaladas serían aún peores. 
de comienzos de los setenta. El efecto positivo como reflejo del control de la inflación a partir de la convertibilidad se pierde completamente hacia 2001, de modo que la devaluación de 2002 no puede sino establecer un nuevo mínimo histórico: el poder adquisitivo del ingreso laboral representó la mitad del vigente a comienzos de los setenta. A partir de allí éste presenta un importante crecimiento, aunque apenas alcanza para recuperar la caída de la devaluación, llegando por tanto a un nivel de $75 \%$ respecto del momento de referencia.

En Estados Unidos el devenir del ingreso laboral real también muestra un quiebre hacia mediados de los años setenta, aunque se trata "sólo" de una desaceleración del crecimiento, tal que hacia 2010 presenta respecto a dicho momento un nivel 35\% mayor. De esta forma, se verifica en este país el proceso esperable de un crecimiento del ingreso laboral real de menor intensidad que el de la productividad.

Si hiciéramos caso omiso del carácter mundial de la producción social en el capitalismo, afirmaríamos que en Argentina este proceso se verificó parcialmente, toda vez que la productividad muestra una tendencia de largo plazo positiva. Ahora bien, lo que hemos intentado poner de manifiesto a partir de la comparación con Estados Unidos es el alejamiento promedio de nuestro país respecto de las condiciones sociales medias de producción, presente desde comienzos de la serie y reforzado profundamente a partir de mediados de los años setenta. En consecuencia, el profundo deterioro del salario real no pareciera poder explicarse por los "incrementos de productividad no transferidos a salarios”. De aquí la pregunta planteada en la introducción: ¿cuál es el papel que desempeña dicho deterioro en la unidad del proceso de acumulación de Argentina? Abordamos esta pregunta en las siguientes dos secciones.

\section{EL DETERIORO DEL INGRESO LABORAL REAL COMO FUENTE EXTRAORDINARIA DE PLUSVALOR}

Hasta aquí, en términos conceptuales se ha considerado que los trabajadores perciben un ingreso laboral acorde al valor de su fuerza de trabajo. Sin embargo, lo cierto es que el conjunto de los capitalistas cuenta con el pago de la fuerza de trabajo por debajo de su valor como fuente adicional de plusvalía, lo que en rigor constituye una fuente extraordinaria de plusvalía, adicional a las plusvalías "normal" u "ordinaria"19. Frente a esto, la pregunta que se impone

19 La generalización de esta condición atenta contra la propia reproducción del sistema (toda vez que no permite la reproducción de la fuerza de trabajo al menos con los atributos productivos que en el momento porta), pero la posibilidad, en cuanto tal, está. 
aquí es evidente: ¿`se ha constituido el deterioro del salario real en una fuente extraordinaria de plusvalía en nuestro país?

En primer lugar, bien podría plantearse que el deterioro en cuestión refleja una caída del valor de la fuerza de trabajo aún mayor que la provocada por la reducción del valor de las mercancías que deben consumir. Ahora bien, este argumento sólo es sostenible si se considera un proceso de descalificación masiva de la fuerza de trabajo. Si bien es cierto que un sector de la misma ha sido víctima del despojo de sus capacidades productivas, otro tanto ha adquirido los atributos de la población trabajadora en escala mundial. Dadas estas dos tendencias contrapuestas, difícilmente el promedio de ellas explique tamaña reducción en el poder de compra asalariado. En este sentido, la evolución de la relación salario-productividad en nuestro país ha sido marcadamente inferior a la de Estados Unidos. ${ }^{20}$

En segundo lugar, podría pensarse que el nivel del salario real a inicios de la década de los setenta se encontraba por encima de su valor, de modo que la reducción observada desde allí en realidad responde a un "ajuste" del salario al verdadero valor de la fuerza de trabajo. Ahora bien, como planteamos previamente, hasta comienzos de los ańos setenta el salario real muestra una evolución compatible con el proceso económico de nuestro país, donde la creciente industrialización requería un obrero colectivo más calificado y, por tanto, con mayor capacidad de consumo.

En ese marco, si la marcada caída del ingreso laboral real no está reflejando ni una caída del valor de la fuerza de trabajo ni un "ajuste" a su valor, entonces tal deterioro del poder adquisitivo representa el pago a una fuerza de trabajo que se vende por debajo de su valor. En este sentido, ¿cuál es el peso que el pago de la fuerza de trabajo por debajo del valor tiene en el plusvalor total, y en el valor anual creado?

Para lograr una cuantificación aproximada "de mínima" de esta fuente extraordinaria de plusvalía y su evolución, se estima para cada año del lapso 1976-2010 la masa salarial que se hubiera verificado si el ingreso laboral real hubiera mantenido año con año su poder adquisitivo de $1970,{ }^{21}$ sería así la diferencia entre la masa salarial hipotética en cada año y la efectivamente observada lo que constituye la plusvalía "extraordinaria" en cuestión, que a su vez

20 En esta línea, Iñigo (2007) comparara la evolución del salario real del obrero industrial de planta de ambos países, esto es, de un trabajador con evolución similar del valor de su fuerza de trabajo, encontrando un retroceso absoluto y relativo del obrero argentino, situación de la cual se deduce la venta de la fuerza de trabajo por debajo de su valor en nuestro país.

21 La elección de dicho año se fundamenta en lo expresado en la nota al pie 19. 
permite distinguir dentro la plusvalía efectiva total y la plusvalía "ordinaria." Además, a modo de control se realizó idéntico ejercicio considerando la masa salarial que se hubiera verificado si el ingreso laboral real hubiera mantenido año con año la relación observada de la evolución entre la productividad y el ingreso laboral real estadounidense. Para fines prácticos, identificamos ambos métodos como I y II, respectivamente. En la gráfica 5 expresaron los resultados, donde los valores negativos reflejan el hecho de que el salario real de los años en cuestión es, en el primer caso, mayor al de 1970 y, en el segundo, mayor al que reflejaría la evolución de la relación entre salario real y productividad estadounidense.

Dado que la participación de la plusvalía en el ingreso es el reflejo invertido de la de los ingresos laborales, carece de sentido detenerse en su evolución. Lo que interesa aquí es notar que, en el marco de que dicha participación presenta desde 1976 un nivel relativamente estable (50/55\%), a la par que un "escalón" por encima de la IsI (40/45\%), la plusvalía extraordinaria en cuestión muestra una participación creciente en el tiempo, tanto en el ingreso total como en la plusvalía efectiva.

Más específicamente, lo que puede observarse es que luego de cada "pico" de su participación, esta fuente extraordinaria de plusvalía se ubica en un nivel promedio superior al anterior, tanto en relación con el ingreso total como con la plusvalía efectiva. Así, dicha fuente significó entre 5 y $10 \%$ del ingreso total hasta la hiperinflación de 1989, entre 10 y 15\% durante la convertibilidad, y 15 y $25 \%$ durante la primera década del siglo xxi. Por su parte, en relación con la plusvalía efectiva su participación aproximada en cada uno de dichos tres lapsos temporales fue de entre 10 y $20 \%$; entre 15 y $30 \%$, y, finalmente, entre 30 y 40 por ciento. ${ }^{22}$

En resumidas cuentas, el pago de la fuerza de trabajo por debajo de su valor se ha instalado, desde la irrupción de la dictadura militar y de modo creciente, como una condición del proceso de acumulación en nuestro país, independientemente de las diferentes formas políticas que la acumulación de capital ha adoptado desde entonces. logía de estimación de la plusvalía extraordinaria con fuente en el pago de la fuerza de trabajo por debajo de su valor confirma las conclusiones presentadas sobre la base de la primera de ellas. 
Gráfica 5. Participación de la plusvalía efectiva y "extraordinaria" según métodos de estimación en el PIB a precios básicos (líneas). Participación de la plusvalía "extraordinaria" según métodos de estimación en la plusvalía efectiva (barras). Argentina. 1976 -2010 (porcentaje).

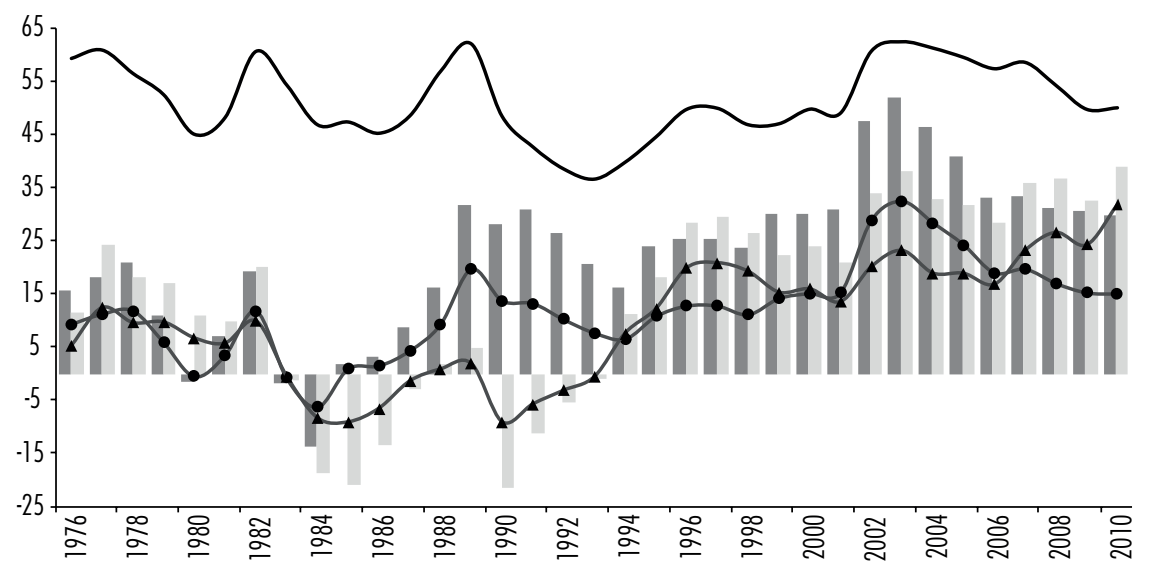

— Plusvalía "extraordinaria" I (IL pod ad 1970) / Plusvalía efectiva

_ Plusvalía "extraordinaria" II (IL según evol prod/IL EU) / Plusvalía efectiva

— Plusvalía efectiva / Ingreso Total

—- Plusvalía "extraordinaria" I (IL pod ad 1970) / Ingreso Total

— Plusvalía "extraordinaria" II (IL según evol prod/IL EU) / Ingreso Total

Fuente: idem, gráfica 1.

\section{CONCLUSIONES A PARTIR DE UNA MIRADA INTEGRADA: EN TORNO A LAS FASES DE ACUMULACIÓN DE CAPITAL EN ARGENTINA}

A lo largo del artículo se ha procurado presentar algunos de los rasgos más sobresalientes del proceso de acumulación de capital de Argentina, partiendo de la mirada conceptual y empírica de la producción social anual en general, y avanzando a partir de desentrañar sus componentes desde el punto de vista de su faceta del ingreso (en tanto refleja el vínculo entre el proceso de producción y la participación económica de la población). Ese camino concluyó planteando que el deterioro del ingreso laboral real ocurrido desde mediados de los ańos setenta implica en nuestro país una creciente fuente extraordinaria de plusvalía. Ahora bien, la pregunta que aún queda pendiente es cuál es el sentido que dicho papel del deterioro del ingreso laboral real tiene en la unidad del proceso de acumulación de nuestro país. Para tales fines, se articulan los distintos puntos nodales desarrollados previamente en un orden particular. 
El capitalismo se distingue como modo de producción por el carácter privado del trabajo, lo que implica que la organización de la producción social se realiza por medio del carácter de valor de los productos del trabajo o, en concreto, de su precio. Justamente por ello constituye un modo de organizar la producción social de contenido mundial, que se realiza bajo la forma de sus fragmentos nacionales. Surge, por tanto, la pregunta en torno a la producción de valor de los países o, en otros términos, de la capacidad de sus valores de uso de representar trabajo social. En este sentido, en la primera sección hemos puesto de manifiesto que desde mediados de la década de los setenta el valor producido por Argentina ha sufrido, primero, un estancamiento, para luego directamente retroceder, de modo que el crecimiento de la posconvertibilidad apenas alcanza para ubicarlo en un nivel similar al de comienzos de la década de los setenta.

Ahora bien, se mostró que la economía argentina no tiene una particular debilidad relativa en lo que respecta a la absorción de ocupación, lo que arroja como resultado una violenta caída de la masa de valor promedio por ocupado. En otros términos, nos enfrentamos con que el trabajo desplegado en promedio en nuestro país es capaz de expresar cada vez menos trabajo social. Tal fenómeno encuentra su fundamento, siempre en términos generales, en el alejamiento sistemático desde entonces de las condiciones sociales medias de producción, como hemos observado en la segunda sección.

Dada la determinación mundial del valor de las mercancías en función de las condiciones sociales de producción, éstas se desarrollan por debajo de tales condiciones medias, para subsistir requieren de fuentes extraordinarias de plusvalía. Más allá del debate en torno a si bajo la Isı la economía nacional presenta o no una productividad menor a la que rige en el mercado mundial, lo cierto es que al menos desde mediados de los setenta los capitales individuales que producen en Argentina requieren, siempre como determinación general, de fuentes extraordinarias de plusvalía que compensen sus mayores costos.

Como consecuencia de la productividad relativamente elevada del trabajo aplicado a los recursos naturales, a nuestro país fluye históricamente desde el resto del mundo renta de la tierra como fuente extraordinaria de plusvalía, que se redistribuye, por distintos mecanismos, a los distintos capitales que producen en su interior (Ińigo, 2007). La magnitud de esta fuente extraordinaria de plusvalía es variable en el tiempo, dependiendo fundamentalmente de los precios de las mercancías exportadas. En segundo lugar, en distintos momentos el endeudamiento externo ha cumplido idéntico papel (Costa et al,2004; Ińigo, 2007). Por sus propias determinaciones de deuda, esta fuente es particularmente endeble, más aún si no se aplica con fines que evidencien 
capacidad de repago. Es en este marco donde cobra sentido el surgimiento e incidencia creciente del pago de la fuerza de trabajo por debajo de su valor como fuente extraordinaria de plusvalor, constituyéndose así en un elemento común de los distintos esquemas económicos adoptados a partir de allí.

Conscientes de que no resulta habitual la introducción de una gráfica en la sección destinada a las conclusiones, creemos que en este caso bien vale la pena la excepción, pues el contenido de la gráfica 6 sintetiza de un modo particularmente claro lo central del artículo. Allí expresamos la masa de valor producida por Argentina (primera sección) desde el punto de vista de los ingresos, combinando la participación de los ingresos laborales en el total (segunda sección) con las tres porciones de la plusvalía: la "extraordinaria" con base en el pago de la fuerza de trabajo por debajo de su valor (tercera sección), la renta de la tierra (tomando los datos de Iñigo, 2007), y la plusvalía "ordinaria" (computada por diferencia).

Como se observa, el estancamiento de la producción de valor de la economía nacional ocurre en conjunción con un profundo retroceso en la capacidad de la economía nacional de producir plusvalía "normal", en tanto que la efectivamente ocurrida se nutre crecientemente de las fuentes "extraordinarias". Y es, a partir de aquí, que creemos que podemos encontrar una posible explicación al devenir del ciclo económico de nuestro país desde, al menos, mediados de los años setenta.

En primer lugar, la baja incidencia de la renta de la tierra y del pago de la fuerza de trabajo por debajo de su valor, conjuntamente con las restricciones al endeudamiento externo determinan el estancamiento de la producción en los años ochenta, marco en el cual se manifiestan profundas tensiones macroeconómicas. En segundo lugar, el deterioro del ingreso laboral real conjuntamente con una explosión del endeudamiento externo le dan cuerpo al crecimiento evidenciado durante la década de los noventa que, como planteamos, comienza a resquebrajarse para finalmente derrumbarse, justamente como resultado, primero, del agotamiento en la capacidad de endeudarse y, luego, de su restricción plena, lo que concluyó en la peor crisis económicosocial de nuestro país. Finalmente, la fase expansiva de la primera década del siglo Xxi, habitualmente presentada como resultado de un "nuevo patrón de crecimiento", tiene en su base tanto un crecimiento de la renta de la tierra (consecuencia de los precios récords de las commodities) como la profundización (viabilizada por la referida crisis) de la fuente extraordinaria con base en el pago de la fuerza de trabajo por debajo de su valor.

Obviamente, no pretendemos con lo anterior haber agotado el análisis respecto de las particularidades del proceso económico de Argentina. Muy 
Gráfica 6. Masa de ingresos laborales, plusvalía "extraordinaria" (según constancia del poder adquisitivo del ingreso laboral en 1970), renta de la tierra y plusvalía "ordinaria" (excluyendo renta de la tierra). Argentina, 1935-2010. (millones de pesos con capacidad de representar valor constante de 1993).

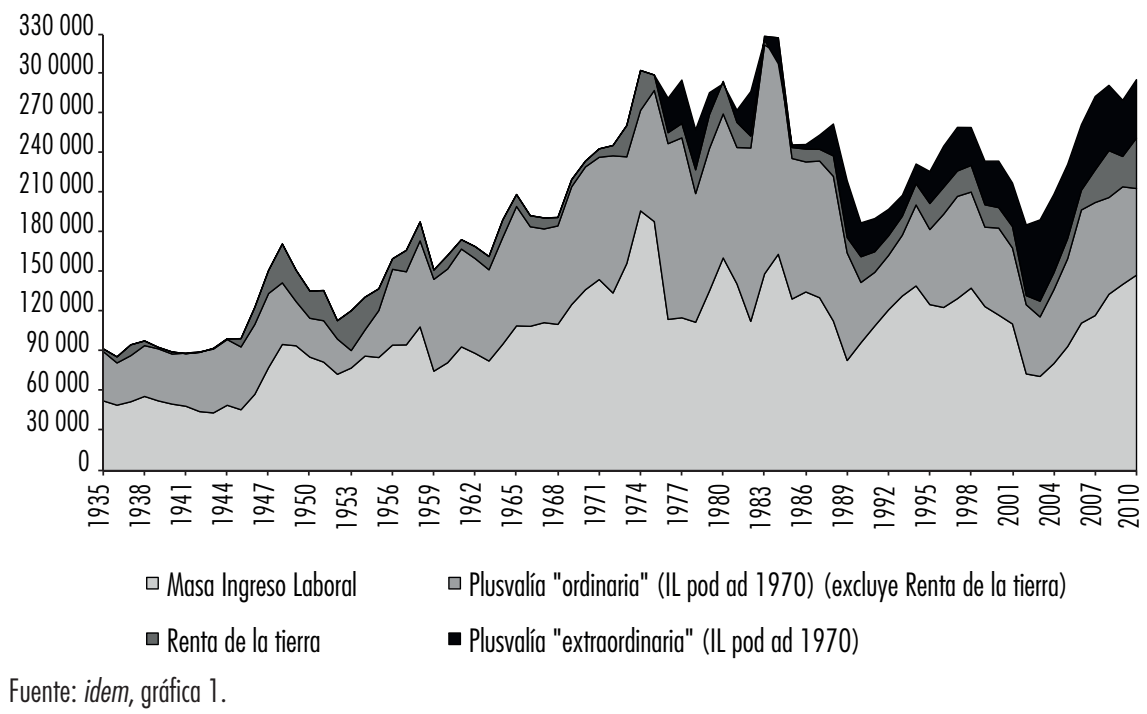

por el contrario, creemos que más bien debe ser considerado como punto de partida de diversas investigaciones futuras. Ahora bien, lo que parece cierto es que no se han generado en nuestro país bases genuinas para el sostenimiento de un sólido proceso de acumulación que permita la mejora en las condiciones de vida de la población. La evolución de la productividad aparece como el aspecto clave a ser considerado. Dilucidar las razones de su evolución pasada, así como las acciones a emprender para mejorar la futura, se impone como una de las principales líneas de investigación por desarrollar.

\section{AGRADECIMIENTOS}

Una versión previa, realizada conjuntamente con Juan M. Graña, fue presentada en el V Congreso alap, Montevideo, Uruguay (del 23 al 26 de octubre de 2012). Agradezco los comentarios realizados en dicha instancia por Andrea Vigorito, así como también a los efectuados a la versión actual del artículo por Carla Borroni, Juan M. Graña y Pilar Piqué. 


\section{BIBLIOGRAFÍA}

Arceo, Nicolás, Ana Paula Monsalvo, y Andrés Wainer (2007), "Patrón de crecimiento y mercado de trabajo: Argentina en la post-Convertibilidad", Realidad Económica, núm. 226, Buenos Aires, IADE, febrero-marzo, pp. 25-57.

Basualdo, Eduardo (2006), Estudios de historia económica argentina. Desde mediados del siglo XX a la actualidad, Buenos Aires, FLACso/Siglo XXI Eds.

Banco Central de la República Argentina (1975), Sistema de cuentas del producto e ingreso de la Argentina, volumen 1: Metodología y fuentes, Buenos Aires, BCRA.

CePal (1988), Estadisticas de corto plazo de la Argentina: cuentas nacionales, industria manufacturera y sector agropecuario pampeano, vol. I, documento de trabajo 28, Buenos Aires, CEPAL.

(1991), Proyecto revisión de las cuentas nacionales y de la distribución del ingreso; informe final, Buenos Aires, CEPAL.

(2010), Anuario estadístico de América Latina y el Caribe, Santiago de Chile CEPAL.

Centro de Investigación y Formación de la República Argentina (CIFRA) (2012), Informe de Coyuntura, núm. 11, Buenos Aires, CifrA, noviembre.

Costa, Augusto; Axel Kicillof, y Cecilia Nahón (2004), "Las consecuencias económicas del Sr. Lavagna. Dilemas de un país devaluado", Realidad Económica, núm. 203, Buenos Aires, IADE, abril-mayo, pp. 70-100.

Diamand, Marcelo (1972), "La estructura productiva desequilibrada argentina y el tipo de cambio", Revista Desarrollo Económico, vol. 12, núm. 45, Buenos Aires, IDES, abril-junio, pp. 25-47.

Fernández Enguita, Mariano (1985), "El problema del trabajo productivo", Revista española de investigaciones sociológicas, núm. 30, Madrid, Centro de Investigaciones Sociológicas, segundo trimestre, pp. 93-148.

Ferrer, Aldo (2004), La economia Argentina. Desde sus orígenes hasta principios del siglo XXI, Buenos Aires, Fondo de Cultura Económica.

Ferreres, Orlando (2005), Dos siglos de economía argentina (1810-2004). Historia argentina en cifras, Buenos Aires, Fundación Norte y Sur/Edit. El Ateneo.

Frenkel, Roberto, y Martín Rapetti (2004), Políticas macroeconómicas para el crecimiento y el empleo, Santiago de Chile, ort.

Graña, Juan Martín (2013), Las condiciones productivas de las empresas como causa de la evolución de las condiciones de empleo. La industria manufacturera en Argentina desde mediados del siglo XX, tesis doctoral (en Ciencias Económicas con mención en Economía), Buenos Aires, Universidad de Buenos Aires. 
Graña, Juan Martín, y A. Lavopa (2008), "15 años de EPH, una serie. Empalme entre sus versiones, Puntual y Continua, 1992-2006”, Documentos de trabajo, núm. 11, CEPED-IIE-FCE-UBA, Buenos Aires.

Graña, Juan Martín, y Damián Kennedy (2008a), 'La unidad de la producción social, punto de partida y objetivo del conocimiento", I Jornadas internacionales de investigación y debate político, Buenos Aires, ceics, del 30 de octubre al 1 de noviembre.

(2008b), Salario real, costo laboral y productividad. Argentina 19472006. Análisis de la información y metodología de estimación, documento de trabajo 12, Buenos Aires, CEPED-IIE-FCE-UBA, noviembre.

Guerrero, Diego (1990), "Cuestiones polémicas en torno a la teoría marxista del trabajo productivo", Politica y Sociedad, núm. 5, Madrid, Universidad Complutense de Madrid, primer cuatrimestre, pp. 119-130.

Iñigo Carrera, Juan (2007), La formación económica de la sociedad argentina, vol. I. Renta agraria, ganancia industrial y deuda externa. 1982 - 2004, Buenos Aires, Imago Mundi.

(2004), El capital: razón histórica, sujeto revolucionario y conciencia, Buenos Aires, Imago Mundi.

Kennedy, Damián (2012), Economia política de la contabilidad social. Vinculos entre la teoría de la riqueza social y sus formas de cuantificación, tesis doctoral (doctorado en Ciencias Económicas con mención en Economía), Buenos Aires, Universidad de Buenos Aires.

Kennedy, Damián, y Juan Martín Graña (2010), "El empobrecimiento de los trabajadores argentinos. Discutiendo sus causas en perspectiva internacional", Revista Pecunia, núm. 10, Facultad de Ciencias Económicas y Empresariales, Universidad de León, primer semestre, pp. 231-263.

Lindenboim, Javier; Juan Martín Grańa, y Damián Kennedy (2005), Distribución funcional del ingreso en Argentina. Ayer y hoy, documento de trabajo 4, Buenos Aires, CEPED-IIE-FCE-UBA.

Lindenboim, Javier; Damián Kennedy, y Juan M. Graña (2010), "La relevancia del debate sobre la distribución funcional del ingreso", Desarrollo Económico, vol. 49, núm. 196, Buenos Aires, IDEs, enero-marzo, pp. 541-571.

Llach, Juan, y Carlos Sánchez (1984), Los determinantes del salario en Argentina. Un diagnóstico de largo plazo y propuestas de políticas, Estudios núm. 29 año VII, Córdoba, IRREAL.

Marx, Karl (2005) [1857-1858], Elementos fundamentales para la crítica de la Economía Politica (Grundrisse) 1857-1858, vol. 1, México D.F., Siglo XxI Editores. 
(1995) [1867], El capital. Crítica de la economía politica, T. I, México D.F., Fondo de Cultura Económica. (2000) [1885], El capital. Crítica de la Economía Politica. T. II, México D.F., Fondo de Cultura Económica.

Monteverde, Ernesto, y Norberto Sallaberry (1981), Cuentas Nacionales. Nociones, documento 14, Buenos Aires, BCRA.

Müller, Alberto (1998), Economía descriptiva. Nociones de cuentas nacionales e indicadores socio-económicos, Buenos Aires, Catálogos.

Naciones Unidas (1993), Sistema de Cuentas Nacionales. Rev. 4, Nueva York, Naciones Unidas.

Propatto, Juan Carlos (2004), El Sistema de Cuentas Nacionales. Visión desde la economía aplicada, Buenos Aires, Ediciones Macchi.

Ricardo, David (2004) [1817], Principios de economía politica y tributación, México D.F., Fondo de Cultura Económica.

Secretaría de Asuntos Económicos (1955), Producto e Ingreso de la República Argentina en el periodo 1935-1954, Buenos Aires, BCRA.

Schvarzer, Jorge, y Andrés Tavosnaska (2008), Modelos macroeconómicos en la Argentina: del 'stop and go' al 'go and crush', documento de trabajo 15, Buenos Aires, CESPA-UBA, marzo.

Shaikh, Anwar (1984), "Cuentas de ingreso nacional y categorías marxistas", Revista Economía, Teoría y Práctica (Primera Época), núm. 4, México DF, Universidad Autónoma Metropolitana - Unidad Iztapalapa, invierno, pp. 3-58.

Shaikh, Anwar, y Ahmet Tonak (1994), Measuring the wealth of nations: The political economy of national accounts, Cambridge, Cambridge University Press.

Smith, Adam (1997) [1776], Investigación sobre la naturaleza y causas de la riqueza de las naciones, México D.F., Fondo de Cultura Económica.

Walras, Leon (1987) [1874], Elementos de economía politica pura (o teoría de la riqueza social), Madrid, Alianza Editorial.

\section{Bases de datos}

Bureau of Economic Analysis (BEA) - Estados Unidos, <http://www.bea.gov/>. Bureau of Labor Statistics (BLS) -Estados Unidos, <http://www.bls.gov/>. Instituto Nacional de Estadísticas y Censos-Argentina, <http://www.indec. gov.ar/>

- Encuesta Permanente de Hogares (ЕPH).

- Cuentas Nacionales.

- Índice de Precios al Consumidor (IPC). 